\title{
ARTICLE
}

\section{Extended X-ray absorption fine structure study on gel/liquid extraction of f-block elements}

\author{
Masahiko Nakase $^{\mathrm{a}^{*}}$, Toru Kobayashi ${ }^{\mathrm{a}}$, Hideaki Shiwaku ${ }^{\mathrm{a}}$, Takuya Kawamura ${ }^{\mathrm{b}}$, Kenji Takeshita ${ }^{\mathrm{b}}$, \\ Tomoo Yamamura ${ }^{\mathrm{c}}$ and Tsuyoshi Yaita ${ }^{\mathrm{a}}$ \\ ${ }^{a}$ Japan Atomic Energy Agency, 1-1-1 Kouto, Sayo-cho, Sayo-gun, Hyogo 679-1578, Japan; ${ }^{b}$ Tokyo Institute of Technology, \\ Laboratory for Advanced Nuclear Energy, 2-12-1 Ookayama, Meguro-ku, Tokyo, 152-8550, Japan; ${ }^{c}$ Tohoku University, 2-1-1 \\ Katahira, Aoba-ku, Miyagi 980-8577, Japan
}

Gel/liquid extraction was investigated to achieve selective recognition and separation of trivalent actinides over lanthanides. $N, N, N^{\prime}, N^{\prime}$-tetraallylpyridine-2,6-dicarboxamine (tetraallyl-PDA) was synthesized and polymerized with $N$-isopropylacrylamide (NIPA) and $N, N^{\prime}$-methylenebisacrylamide (BIS) to produce a thermosensitive hydrogel. Introducing ligands into a three-dimensional network of thermosensitive hydrogel is expected to allow the complex structure and coordination behavior to be controlled. The PDA is an $N, O$ hybrid donor ligand and typical PDA derivatives are $O$-donor-driven and show higher extractability for heavier lanthanides. Tetraallyl-PDA showed moderate extractability under weakly acidic conditions with a series of lanthanides, and its extractability for heavier lanthanides was higher owing to the stronger surface charge of the heavier lanthanides. NIPA gel swells and shrinks more effectively under weakly acidic conditions. Hence, tetraallyl-PDA was applicable for gel/liquid extraction. To understand the difference in complexation between the solution and gel, the radial structural function (RSF) was obtained by extended X-ray absorption fine structure (EXAFS) experiments at Super Photon Ring-8 GeV (SPring-8). The RSF of solution with gels with and without ligands at different temperatures showed slight differences. As the temperature increased, the RSF decreased owing to the increase in thermal vibration, but PDA-NIPA-BIS gel showed a shift in the first peak toward the shorter direction. This shift was attributed to the change in stoichiometry of PDA-Ln(III) complexes or coordination number of water molecules affected by the change of conformation of polymer chains and the hydrophilic/hydrophobic properties in the hydrogel. Theoretical fitting was difficult because various chemical species coexist in gel system. Future work will include further fitting, optimizing the gel synthesis, and modifying the ligands for practical use.

Keywords: Extended X-ray absorption fine structure; radial structural function; lanthanides; gel/liquid extraction; thermosensitive gels

\section{Introduction}

Selective recognition and separation of trivalent actinides over lanthanides by solvent extraction is difficult owing to the chemical similarity of these species [1,2]. Particularly in nuclear reprocessing, separating trivalent actinides from lanthanides is important to minimize the burden of final disposal. The ionic radii of trivalent $\mathrm{Am}$ and $\mathrm{Eu}$ and their properties in solution are similar. Solvent extraction is a conventional method for metal separation (Figure 1(a)), and is used for continuous large-scale separation. However, under some conditions, a third phase that is immiscible with the aqueous and organic phases is produced that disrupts stable operation. Furthermore, during highly radioactive processes, such as nuclear fuel reprocessing, organic solvent tends to form secondary radioactive waste and is a source of unwanted radical species [3-6]. Extraction

*Corresponding author. Email: nakase.masahiko@jaea.go.jp chromatography also has been studied extensively for effective separation of trivalent actinides from lanthanides. In extraction chromatography, ligands usually used in solvent extraction are immobilized physically on porous silica or resins [7-12]. Thanks to the nature of this type of chromatography, a high number of theoretical plates is expected and the immobilized ligand allows the extractability and selectivity to be tuned. However, the ligands can be eluted after reuse and depend on the solution. To solve this problem, the ligands can be chemically immobilized on a polymer [13-15]. By introducing ligands to thermosensitive gel (Figure 1(b)), the extraction behavior can be controlled by changing the gel conformation through temperature or $\mathrm{pH}$ without using an organic phase. In solvent extraction, extractability depends on many factors such as the relative permittivity, thermodynamics, and extraction equilibrium. In gel/liquid extraction, more factors other than solvent 
extraction also affect the adsorption behavior, including steric hindrance by polymer chains, the local water structure confined by the polymer chains, and physicochemical properties, such as water content, swelling properties, and thermo-sensitivity. Takeshita et al. synthesized selective ligands for f-block elements, such as $N, N, N^{\prime}, N^{\prime}$-tetrakis(2-methylpyridyl) ethylene -diamine (TPPEN), and introduced the ligand into hydrogels. Some hydrogels were synthesized as uniform, thin coatings on porous silica to allow quick responses to external stimuli. Am(III)/Eu(III) separation by TPPEN-NIPA gel was conducted by changing the temperature; Am(III) was adsorbed at low temperature in swelling state more significantly and not extracted much at high temperature, where $\mathrm{Eu}(\mathrm{III})$ was not extracted much in both temperature [16]. However, the fundamental mechanism is still not known. Therefore, extractability and structural study were conducted with $\mathrm{Nd}(\mathrm{III})$ and $\mathrm{Eu}(\mathrm{III})$ before experiments with actual actinide and a series of lanthanide. Investigation of the complexation of the difference between in solution and gel will lead practical use of the gel/liquid extraction technique.

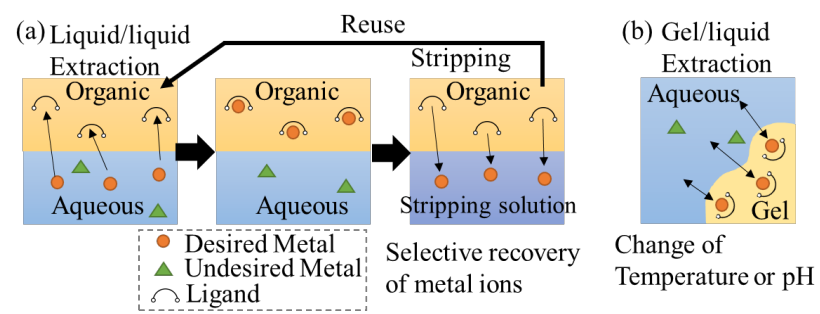

Figure 1. Schematic of (a) liquid/liquid and (b) gel/liquid extraction.

\section{Experimental}

$N, N, N^{\prime}, N^{\prime}$-tetraallylpyridine-2,6-dicarboxamine (tetra allyl-PDA) was synthesized as follows. 2,6-Pyridine dicarbonyl dichloride (1 g, $3.07 \mathrm{mmol}$; Sigma-Aldrich) was dissolved in chloroform $(10 \mathrm{~mL})$ in a $100 \mathrm{~mL}$ flask. Diallylamine (2 equiv; Wako Chemicals) and triethyl -amine (2.5 equiv; Wako Chemicals) were added dropwise at $5{ }^{\circ} \mathrm{C}$. The mixture was stirred for $24 \mathrm{~h}$ at room temperature. The mixture was washed three times with $1 \mathrm{M} \mathrm{HCl}$ solution and with distilled water, dried over sodium sulfate (Wako Chemicals), and the chloroform was evaporated to give the product as a liquid (0.8 g, $2.46 \mathrm{~mol}, 80.1 \%$ yield). ${ }^{1} \mathrm{H}$ NMR (400 $\mathrm{MHz}$ ) spectra was recorded on a spectrometer (400MR, Varian) at $20{ }^{\circ} \mathrm{C}$ in $\mathrm{CDCl}_{3}$ with tetramethylsilane as the internal standard: ${ }^{1} \mathrm{H}-\mathrm{NMR}\left(400 \mathrm{MHz}, \mathrm{CDCl}_{3}\right): \delta 7.89(\mathrm{t}$, $1 \mathrm{H}, J=7.86 \mathrm{~Hz}), 7.69(\mathrm{~d}, 2 \mathrm{H}, J=7.86 \mathrm{~Hz}), 5.87(\mathrm{ddt}$, $2 \mathrm{H}, J=17.28,10.08,5.80 \mathrm{~Hz}), 5.79(\mathrm{ddt}, 2 \mathrm{H}, J=17.16$, $10.28,5.64 \mathrm{~Hz}), 5.26(\mathrm{dd}, 2 \mathrm{H}, J=17.28,1.30 \mathrm{~Hz}), 5.24$ (dd, $2 \mathrm{H}, J=10.08,1.30 \mathrm{~Hz}), 5.13(\mathrm{dd}, 2 \mathrm{H}, J=10.28$, $1.20 \mathrm{~Hz}$ ), $5.10(\mathrm{dd}, 2 \mathrm{H}, J=17.16,1.20 \mathrm{~Hz}), 4.15(\mathrm{~d}, 4 \mathrm{H}$, $J=5.80 \mathrm{~Hz}), 3.98$ (d, 4H, $5.64 \mathrm{~Hz})$; chemical formula: $\mathrm{C}_{19} \mathrm{H}_{23} \mathrm{~N}_{3} \mathrm{O}_{2}$; molecular weight: 325.41 .

Solvent extraction was performed with tetraallyl
-PDA diluted in chloroform, and a series of lanthanides (Ln) from $\mathrm{La}$ to $\mathrm{Lu}$, except $\mathrm{Pm}$, in nitric acid. The aqueous phase was $0.5 \mathrm{mmol} / \mathrm{L}$ solution of each $\mathrm{Ln}$ (total of $7 \mathrm{mmol} / \mathrm{L}$ ) in $0.001 \mathrm{~mol} / \mathrm{L}$ nitric acid and the organic phase was $100 \mathrm{mmol} / \mathrm{L}$ tetraallyl-PDA in chloroform. The phases $(1 \mathrm{~mL}$ each) were shaken together vigorously in $6 \mathrm{~mL}$ vials for $3 \mathrm{~h}$, and then centrifuged to separate the phases. The concentration of the metal ions in the aqueous phase was measured by inductively coupled plasma optical emission spectroscopy (SPS3500, SII) and evaluated by Eq. (1).

$$
\% E=\frac{C_{\mathrm{aq}, \text { initial }}-C_{\mathrm{aq}, \text { after }}}{C_{\mathrm{aq} \text {,initial }}} \times 100
$$

Here, $\% E$ is the percent extraction and $C$ is the metal ion concentration. Ligand immobilized $N$-isopropylacrylamide (NIPA) gel was synthesized by the following procedure. $0.8 \mathrm{mmol} / \mathrm{mL}$ NIPA, 0.4 $\mathrm{mmol} / \mathrm{mL} N, N^{\prime}$-methylenebisacrylamide (BIS), and 0.4 $\mathrm{mmol} / \mathrm{mL}$ tetraallyl-PDA (Figures 2(a)-(c)) were dissolved in $N, N$-dimethylformamide (DMF; total solution volume of $500 \mu \mathrm{L}$ ) in a narrow Pyrex tube. The stoichiometric ratios of the polymerizable functional groups of crosslinkers and monomers were not considered because the focus of this study was not optimizing the synthesis, but comparing the radial structural function (RSF) by EXAFS. The concentration of the radical initiator, 2,2'-Azoisobutyronitrile (AIBN) was $1 \mathrm{~mol} \%$ of the monomer. After vigorous bubbling with nitrogen for $5 \mathrm{~min}$, the solution was heated to $70{ }^{\circ} \mathrm{C}$ and kept in an oven overnight for radical polymerization. The structure of PDA-NIPA-BIS gel is shown in Figure 2(d).

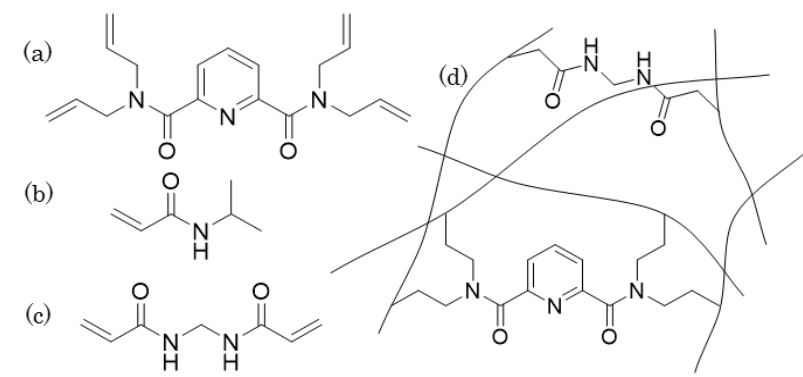

Figure 2. Structures of (a) tetraallyl-PDA, (b) NIPA, (c) BIS, and (d) NIPA-BIS-PDA gel.

Figure 3 shows photographs of the preparation of gel samples for EXAFS experiments. The gels were removed from the Pyrex tube, ground by a pestle and mortar, and washed with distilled water several times to remove DMF and unreacted chemicals. The dry gel (3 $\mathrm{mg}$ ) was placed in a $6 \mathrm{~mL}$ vial, $0.1 \mathrm{~mol} / \mathrm{L} \mathrm{Nd}\left(\mathrm{NO}_{3}\right)_{3}$ or $\mathrm{Eu}\left(\mathrm{NO}_{3}\right)_{3}$ and $0.001 \mathrm{~mol} / \mathrm{L} \mathrm{HNO}_{3}$ (adsorption solution) were added, and the mixture was left overnight. The adsorption solution was replaced with $0.001 \mathrm{~mol} / \mathrm{L}$ $\mathrm{HNO}_{3}$ and the sample was washed to remove free $\mathrm{Nd}\left(\mathrm{NO}_{3}\right)_{3}$ or $\mathrm{Eu}\left(\mathrm{NO}_{3}\right)_{3}$ solution. The wet gels were transferred to a quartz cell (light path length: $10 \mathrm{~mm}$ ). The sample temperature was kept at $5-60{ }^{\circ} \mathrm{C}$ by a 
temperature-controlled water circulation system because the phase transfer temperature of NIPA-Bis gel is about $37{ }^{\circ} \mathrm{C}$, and the temperature in the quartz cell was monitored remotely. The EXAFS experiments were done at SPring-8 on beamline BL11XU (2016A3504 and 2016B3508). The EXAFS spectra of $\mathrm{Nd}(\mathrm{III})$ and $\mathrm{Eu}(\mathrm{III})$ in gel and solution were measured with K-edges in transmission mode. Data manipulation was performed with $W$ in $X A S$ version 2.3 [17].
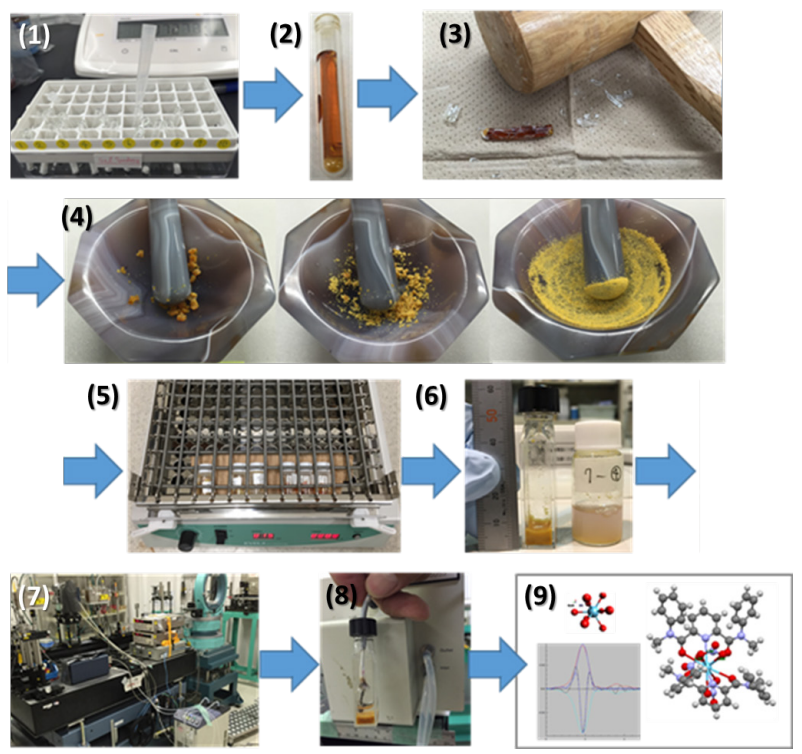

Figure 3. Preparation of gel samples for EXAFS experiments. From top left to bottom right: (1)mixing chemicals, (2)polymerization, (3)removal from glass tube, (4)grinding samples, (5)washing and drying, (6)adsorption, (7)carrying to beam line, (8)equipment of thermocouple and setting, (9)data acquisition and analysis

\section{Results and discussion}

\subsection{Solvent extraction}

Figure 4 shows the results of solvent extraction. Heavier lanthanides were extracted more effectively owing to their stronger surface charge density. Typically, PDA derivative ligands, such as those with octyl and tolyl groups, show high extractability when the acidity is high [18-21]. However, the tetraallyl-PDA could extract $\mathrm{Ln}$ at lower acidity which allows for $\mathrm{pH}$ or temperature swing adsorption/desorption technique. The swelling property of NIPA-BIS gel tends to be worse in highly acidic condition, hence, the ligand inserted into the hydrogel requires sufficient extractability for the desired metal ions at lower acidity. Therefore, it was confirmed that the tetraallyl-PDA is a suitable ligand to insert into NIPA-BIS gel and to determine the difference in the complex structures in gel and solution. This gel can also extract hexavalent actinide in acidic condition as well, and such adsorption behavior will be reported elsewhere.

\subsection{EXAFS experiments}

The RSF in gel gives the mixed information of free

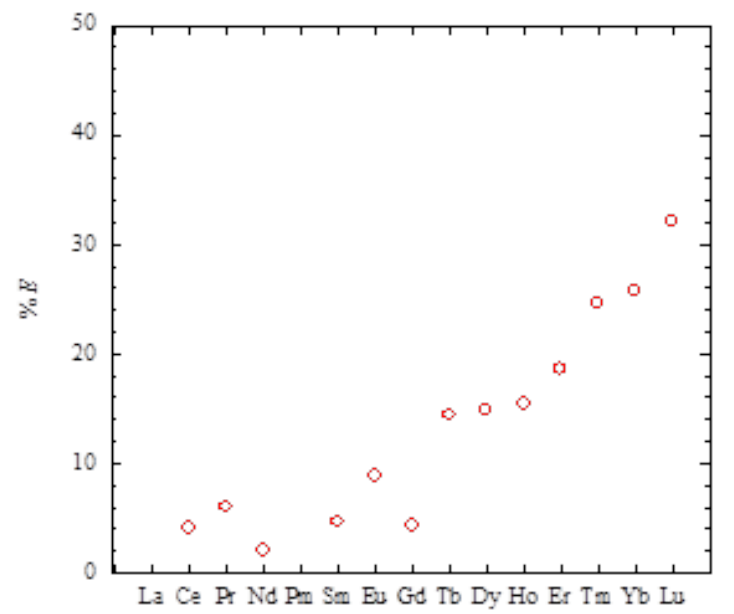

Figure 4. Percent extraction of a series of lanthanides with tetraallyl-PDA in $0.001 \mathrm{M}$ nitric acid.

hydrated $\mathrm{Nd}$ (III) ions and some kinds of complexes which makes the curve fitting difficult. Though a discussion of complex structures only by the RSF is not sufficient, the RSF of $\mathrm{Ln}$ (III) complexes in solution and in gel were simply compared due to this complexity. Figure 5 shows the temperature dependence of the RSFs of $\mathrm{Nd}(\mathrm{III})$ in nitric acid and $\mathrm{Nd}$ (III)-NIPA -BIS-PDA gel. When the temperature of the $\mathrm{Nd}$ (III) solution was increased, the position of the first peak, which corresponds to the $\mathrm{O}$ atoms of hydrated water or nitrates, did not shift but the height of the peak was lowered by the increase in the thermal vibration of molecules. This tendency indicates no structural changes of $\mathrm{Nd}(\mathrm{III})$ hydrated complex. For the Nd(III)-NIPA-BIS -PDA gel, the position of the first peak which corresponds to not only the $\mathrm{O}$ atoms of hydrated water or nitrates, but also the $\mathrm{N}$ and $\mathrm{O}$ atoms of tetraallyl-PDA, was shifted toward the shorter direction as well as the decrease in intensity. This difference indicates a slight change in complex structure such as stoichiometry of tetraallyl-PDA$\mathrm{Nd}(\mathrm{III})$ complexes and the number of hydrated water molecules in the gel. Though the crystal structures of Ln-tetraallyl-PDA have not been acquired yet, the structural studies of PDA with phenyl and methyl groups were reported and $\mathrm{Ln}$ (III) coordination with 3-4 PDA molecules in solvent extraction was suggested [22-25]. In shrinking state of gel at higher temperature than the volume phase transition, the coordination number of tetraallyl-PDA can be decreased because the coordination space around $\mathrm{Nd}(\mathrm{III})$ becomes more crowded and polymer chains interrupt the coordination. Less number of tetraallyl-PDA coordination leads shorter bonding distances of $\mathrm{O}, \mathrm{N}$ atoms of tetraallyl-PDA and $\mathrm{Nd}(\mathrm{III})$. The RSF of gel was not clear enough to discuss the difference in further $\mathrm{R}$ range. To understand the further region, revision of experimental method or other methods are needed. 


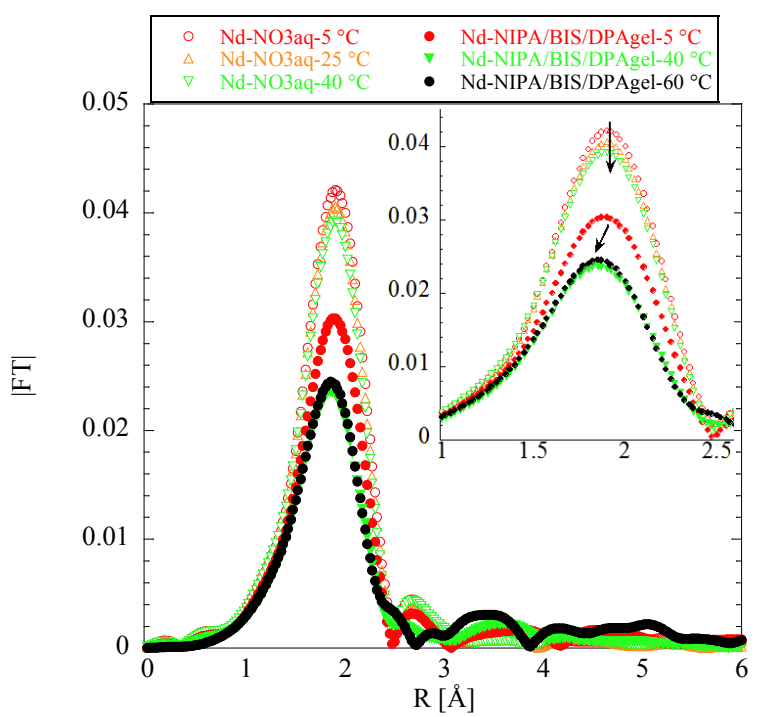

Figure 5. Temperature dependence of RSFs of $\mathrm{Nd}(\mathrm{III})$ in nitric acid and Nd(III)-NIPA-BIS-PDA gel in water.
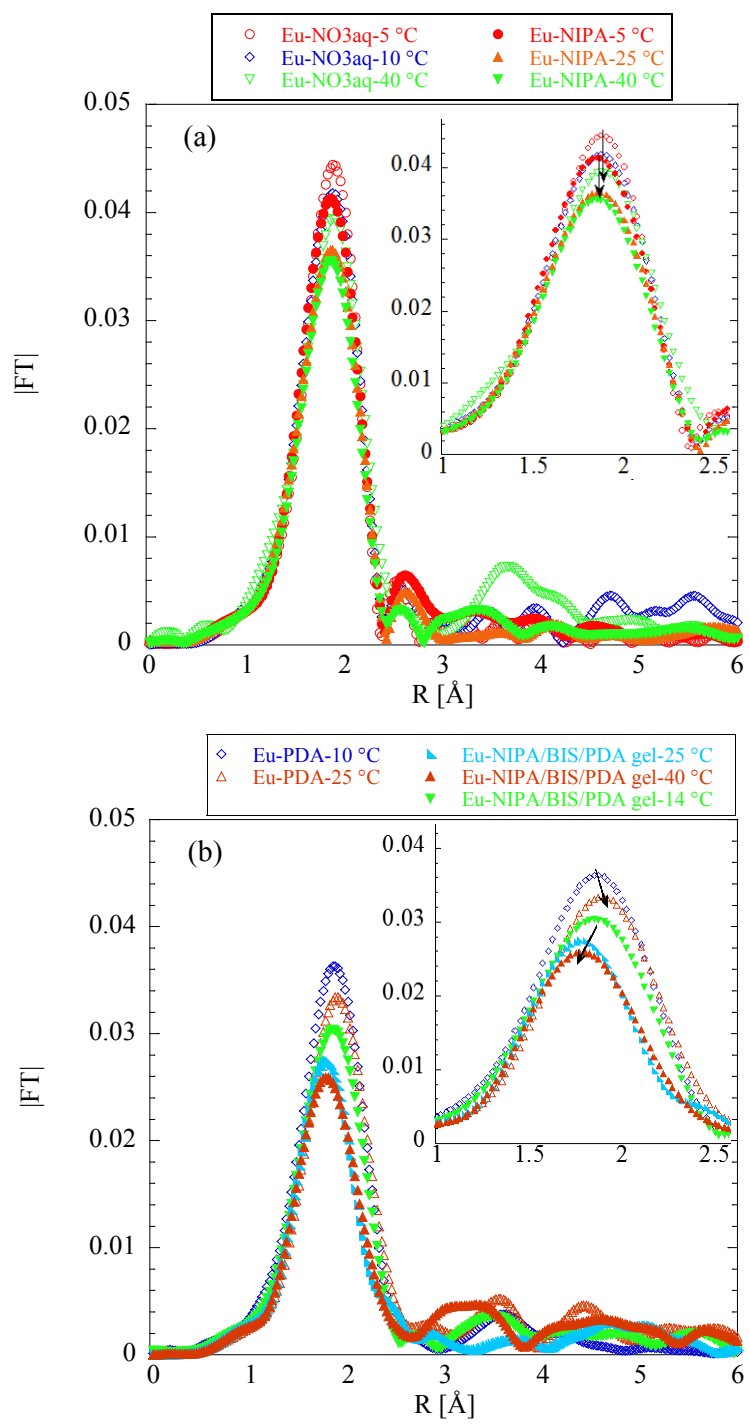

Figure 6. Temperature dependence of RSFs of (a) Eu(III) in nitric acid and Eu(III)-NIPA-BIS-PDA gel and (b) $\mathrm{Eu}(\mathrm{III})-\mathrm{NIPA}-\mathrm{BIS}-\mathrm{PDA}$ gel in water and $\mathrm{Eu}(\mathrm{III})-\mathrm{PDA}$ in methanol.
Figure 6 shows the temperature dependence of RSFs of (a) Eu(III) in nitric acid and Eu(III)-NIPA-BIS-PDA gel and (b) Eu(III)-NIPA-BIS-PDA gel in water and Eu(III)-PDA in methanol. The RSFs of Eu(III) solution showed a similar tendency to $\mathrm{Nd}$ (III) solution case. In both $\mathrm{Nd}(\mathrm{III})$ and $\mathrm{Eu}(\mathrm{III})$ cases, the shift seems not linear according to the temperature change which is similar to the volume phase transition behavior of NIPA gel. By comparison between Eu(III)-NIPA-BIS-PDA gel and $\mathrm{Eu}(\mathrm{III})-\mathrm{PDA}$ in methanol as shown in Figure 6(b), the tendency was different. This indicates the difference in coordination of PDA and $\mathrm{Ln}(\mathrm{III})$ between in organic solvent and in hydrogel. There is also a possibility of monodentate coordination as well as multidentate coordination of tetraallyl-PDA and $\mathrm{Ln}(\mathrm{III})$ in gel and their ratio might be variable. The changes in RSFs with changing in temperature became highlighted by EXAFS experiments, and further discussion will be possible with a comparison of adsorption behaviors of a series of $\mathrm{Ln}(\mathrm{III})$ and change of RSFs with variety of temperature.

\section{Conclusions}

Tetraallyl-PDA was synthesized and polymerized with NIPA and BIS, which produced a thermosensitive gel. In solvent extraction with chloroform as an organic phase and nitric acid as an aqueous phase, the extractability of the heavier lanthanide ( $\operatorname{Ln}(\mathrm{III})$ ) was higher in $0.001 \mathrm{~mol} / \mathrm{L} \mathrm{HNO}_{3}$ solution, which was effective for gel/liquid extraction without sacrificing gel swelling. The RSFs obtained by EXAFS for solution and NIPA-BIS-PDA gel were compared with $\mathrm{Nd}(\mathrm{III})$ and $\mathrm{Eu}(\mathrm{III})$. The NIPA-BIS-PDA gel in both cases of $\mathrm{Nd}$ (III) and $\mathrm{Eu}(\mathrm{III})$ showed shifts in the first peak with increasing temperature, but not for $\mathrm{Nd}(\mathrm{III}), \mathrm{Eu}(\mathrm{III})$ and $\mathrm{Eu}(\mathrm{III})-\mathrm{NIPA}$ in nitric acid. Also, it was suggested that the coordination is different in organic solvent and in gel by comparison of Eu(III)-NIPA-BIS-PDA gel and Eu(III)-PDA in methanol. Theoretical curve fittings of the RSFs were difficult due to the mixture of the complexes in gel; however, the results indicated a slight change in complex structure. Understanding the difference in complexation in solution and gel further should allow the selective recognition and separation of specific f-block elements in the future.

\section{Acknowledgements}

This research was supported by the Japan Society for the Promotion of Science through a Grant-in-Aid for JSPS Fellows (research number: 26-202) and Overseas Research Fellowship (434). EXAFS experiments were conducted at Spring-8 (2106A3054 and 2016B3058).

\section{References}

[1] J.Veliscek-Carolan, Separation of actinides from spent nuclear fuel: A review, J. Hazard Mater. 318 (2016), pp.266-281. 
[2] J.N. Mathur et al., Actinide partitioning - a review. Solvent Extr. Ion Exc. 19 (2001), pp.357-390.

[3] B.J. Mincher et al., Review article: The effects of radiation chemistry on solvent extraction 2: A review of fission-product extraction. Solvent Extr Ion Exc. 27 (2009), pp.331-353.

[4] B.J.Mincher et al., Review article: The effect of radiation chemistry on solvent extraction: 1 . Conditions in Acidic Solution and a Review of TBP Radiolysis, Solvent Extr. Ion Exc. 27 (2009) pp.1-25.

[5] B.J. Mincher et al., Review article: The effect of radiation chemistry on solvent extraction 3: A Review of Actinide and Lanthanide Extraction, Solvent Extr. Ion Exc. 27 (2009), pp.579-606.

[6] B.J. Mincher et al., Review: The effect of radiation chemistry on solvent extraction 4: Separation of the Trivalent Actinides and Considerations for Radiation-Resistant Solvent Systems, Solvent Extr. Ion Exc. 28 (2010), pp.415-436.

[7] M. Arisaka et al., Development of extraction chromatographic adsorbent using alkylpyridine -dicarboxyamides as extractant for separation of trivalent minor actinides from lanthanides stability and separation ability against nitric acid exposure and gamma-ray irradiation, J. Nucl. Sci. Technol. 51 (2014), pp.457-464.

[8] J.A. Drader et al., Assessment of monoamide extractants and solid supports as new extraction chromatographic materials, Sep. Purif. Technol. 163 (2016), pp.352-356.

[9] S. Chappa et al., Spacer monomer in polymer chain influencing affinity of ethylene glycol methacrylate phosphate toward $\mathrm{UO}_{2}{ }^{2+}$ and $\mathrm{Pu}^{4+}$ Ions, Ind. Eng. Chem. Res. 55 (2016), pp.8992-9002.

[10]C.K. Vyas, Distribution behavior of U(VI), Am(III) and $\mathrm{Eu}(\mathrm{III})$ on diglycolamide based extraction chromatographic resin in perchloric acid medium, J. Radioanal. Nucl. Ch. 298 (2013), pp.1643-1650.

[11]Chirag K. VyasPranav et al., Distribution behavior of U(VI), Am(III) and Eu(III) on diglycolamide based extraction chromatographic resin in perchloric acid medium, J. Radioanal. Nucl. Chem. 298 (2013) pp.1643-1650.

[12]E.P. Horwitz et al., Novel extraction of chromatographic resins based on tetraalkyldiglycolamides: characterization and potential applications, Solvent. Extr. Ion. Exc. 23 (2004), pp.319-344.

[13]K. Takeshita, Thermal-swing extraction of $\mathrm{Cd}(\mathrm{II})$ by thermosensitive gel crosslinked with nitrogen-donor ligands, J. Chem. Eng. Jpn. 36 (2003), pp.1253-1258.
[14]K. Takeshita et al., Thermal-swing extraction of cadmium(II) by thermosensitive polymer gel crosslinked with encapsulating hexadentate ligand, Chem. Lett. 36 (2007), pp.1032-1033

[15]Y. Inaba et al., Thermoresponsive extraction of cadmium(II) ions by poly(TPEN-NIPA) gels. Effect of chain length and branched spacer structure on gel formation and extraction behavior, Polym. J. 43 (2011), pp.630-634.

[16]K. Takeshita et al., Separation of Americium(III) and Europium(III) by thermal-swing extraction using thermosensitive polymer gel, Prog. Nucl. Energ. 50 (2008), pp.466-469.

[17]T. Ressler, WinXAS: a Program for X-ray Absorption Spectroscopy Data Analysis under MS-Windows, J. Synch. Rad. 5 (1998), pp.118-122.

[18]A. Paulenova et al., Extraction of lanthanides with diamides of dipicolinic acid from nitric acid solutions I, Separ. Sci. Technol. 43 (2008) pp.2606-2618.

[19]A. Shimada et al., Extraction studies of lanthanide III Ions with $N, N^{\prime}$-Dimethyl- $N, N^{\prime}$-diphenylpyri -dine-2,6-dicarboxyamide (DMDPhPDA) from Nitric Acid Solutions, Solv. Ext. Ion. Exch. 22 (2004), pp.147-161.

[20]A. Shimada et al., Extraction of Am(III) and Lanthanide(III) ions from $\mathrm{HNO}_{3}$ solutions using $N, N$ '-dimethyl- $N, N$ '-diphenylpyridine-2,6-dicarbox yamide, J. Chem. Eng. Jpn. 11 (2004), pp.1-10.

[21]T. Yaita et al., XAFS and spectroscopic characterization for the structure and electronic structure of Ce-DMDPhPDA complexes in Methanol, Phys. Scripta, T115 (2005), pp.302-305

[22]M. Dobler et al., Quantum chemical study of LnIII (pyridine-dicarboxy-amide), Phys. Chem. Chem. Phys. 5 (2003), pp.2499-2504.

[23]P. Devi et al., Synthesis and structural characterisation of amides from picolinic acid and pyridine-2,6-dicarboxylic acid, Sci. Rep-UK 5 (2015), p.9950.

[24]A.Fujiwara et al., Structural studies of lanthanide nitrate- $N, N^{\prime}$-dimethyl- $N, N^{\prime}$-diphenylpyridine-2, 6 -dicarboxyamide complexes, J. Alloy Compd. 456 (2008), pp.429-435.

[25]C. Brouca-Cabarrecq et al, Hydrothermal investigation of the lanthanide $(\mathrm{Ln}=\mathrm{La}, \mathrm{Ce}, \mathrm{Pr}$, $\mathrm{Nd}$, Sm) 2,6-pyridinedicarboxylate system, Inorg. Chim. Acta 332 (2002), pp.54-60. 